\title{
Pacific
}

Journal of

Mathematics

\section{JEAN BOURGAIN'S ANALYTIC PARTITION OF UNITY VIA HOLOMORPHIC MARTINGALES}

\author{
PAUL F.X. MÜLLER
}




\title{
JEAN BOURGAIN'S ANALYTIC PARTITION OF UNITY VIA HOLOMORPHIC MARTINGALES
}

\author{
PAUl F.X. MÜLleR
}

Using stopping time arguments on holomorphic martingales we present a soft way of constructing J. Bourgain's analytic partition of unity. Further applications to Marcinkiewicz interpolation in weighted Hardy spaces are discussed.

1. Introduction. In his 1984 Acta Mathematica paper Jean Bourgain derives new Banach space properties of $H^{\infty}$ and the disc algebra from the existence of the following analytic partition of unity:

THEOREM 1 [J. Bourgain]. Given $f$, a strictly positive integrable function on $\mathbf{T}$ with $\int f(t) d t=1$ and $0<\delta<1$ then, there exist functions $\tau_{j}, \gamma_{j} \in H^{\infty}(T)$ and positive numbers $c_{j}$ such that:

1. $\left\|\gamma_{j}\right\|_{\infty}<C$

2. $\sum\left|\tau_{j}\right|<C$

3. $\left|\tau_{j}\right| f<c_{j}$

4. $\sum c_{j}\left\|\tau_{j}\right\|_{1}<\delta^{-C}$

5. $\int\left|1-\sum \gamma_{j} \tau_{j}^{2}\right| f d t<\delta$.

Here I wish to present a soft way to this construction which results from using probabilistic tools such as holomorphic martingales.

I should like to point out here that a proof for the existence of analytic partitions of unity - much simpler than J. Bourgain's - has been given recently by Serguei Kislyakov. See [K1] and [K2]. In [K3] S. Kislyakov derived J. Bourgain's result on $p$-summing operators from the following weighted Marcinkiewicz decomposition.

THEOREM 2 [S. Kislyakov]. For any positive weight $b$ on $\mathbf{T}$ there exists a weight $B \geq b$ and $\int B d t<C \int b d t$ so that for any $\lambda>$ 
0 and $f \in H^{1}(T, B)$ there exists $g \in H^{\infty}(T)$ and $h \in H^{1}(T, B)$ satisfying:

1. $f=g+h$

2. $\|g\|_{\infty} \leq \lambda$

3. $\int h B d t \leq C \int_{\{|f|>\lambda\}}|f| B d t$.

Up to small perturbations we shall obtain a stochastic version of Kislyakov's decomposition which allows us to prove the following:

TheOREM 3 [J. Bourgain]. For any 2-summing operator $S$ on the disc algebra and any $2<q<\infty$ the $q$-summing norm satisfies the interpolation inequality

$$
\pi_{q}(S) \leq C_{q} \pi_{2}(S)^{\frac{2}{q}}\|S\|^{1-\frac{2}{q}}
$$

A very elegant proof of this interpolation inequality has been given by Gilles Pisier who used vectorvalued $H^{1}$ spaces. See $[\mathbf{P}]$.

2. The main result. Holomorphic martingales were introduced by H. Föllmer in $[\mathbf{F}]$ and N. Varopoulos in [V]. They are stable under stopping times, and generalize analytic functions on the unit circle. This connection has led to probabilistic proofs of several results in Analysis, including Carleson's corona theorem [V], the existence of a logmodolar Banach algebra having no analytic structure $[\mathbf{C}]$ and P.W. Jones's interpolation theorems between $H^{1}$ and $H^{\infty}[\mathbf{M 1}$, [M2].

This paper is not selfcontained! We freely use definitions from [V] without further explanation. $(\Omega, P)$ denotes Wiener's measure space governing complex Brownian motion. $H^{p}(\Omega, P)$ is the subspace of $L^{p}(\Omega, P)$ consisting of holomorphic random variables. (See [V].)

THEOREM 4. Given $\Delta$, a strictly positive integrable function on $(\Omega, P)$ with $\int \Delta d P=1$ and $0<\delta<1$, there exist functions $w_{j}, \theta_{j} \in H^{\infty}(\Omega)$ and positive numbers $c_{j}$ such that:

1. $\left\|\theta_{j}\right\|_{\infty}<C$

2. $\sum\left|w_{j}\right|<C$

3. $\left|w_{j}^{2}\right| \Delta<c_{j}$

4. $\sum c_{j}\left\|w_{j}^{2}\right\|_{1}<\delta^{-C}$ 
5. $\int\left|1-\sum \theta_{j} w_{j}^{4}\right| \Delta d P<\delta$.

Probability offers a soft way of constructing the functions $\theta_{j}$ so that the verification of (5) becomes much easier than in J. Bourgain's proof. See [B1, pp. 11, 12]. The probabilistic concept will be merged with analytic tools, such as Havin's lemma, which we use in the following form, due to J. Bourgain. (See [B1].)

THEOREM 5. For every measurable subset $E$ of $\Omega$ and $0<\epsilon<1$ there exist functions $\alpha, \beta \in H^{\infty}(\Omega)$ such that:

1. $|\alpha|+|\beta| \leq 1$

2. $\left|\alpha-\frac{1}{5}\right|<\epsilon$ on $E$.

3. $|\beta|<\epsilon$ on $E$.

4. $\|\alpha\|_{1}<C|\log \epsilon|^{2} P(E)$

5. $\|1-\beta\|_{2}<|\log \epsilon| P(E)^{\frac{1}{2}}$.

Proof of Theorem 4. We shall first determine a new weight: Let $d=\Delta^{\frac{1}{2}}$ and put

$$
A(d):=\sup _{t}\left|E\left(d \mid \mathcal{F}_{t}\right)\right|
$$

then we let

$$
\Delta_{1}=\sum_{n=0}^{\infty} A^{n}(d)(C 2)^{-n}
$$

where $C$ is determined by Doob's inequality: For $d \in L^{2}(\Omega)$

$$
\int \sup _{t}\left|E\left(d \mid \mathcal{F}_{t}\right)\right|^{2}<C\|d\|_{2}^{2}
$$

Clearly this construction gives,

1. $A\left(\Delta_{1}\right)<\Delta_{1} 3 C$

2. $\Delta^{\frac{1}{2}}<C \Delta_{1}$

3. $\int \Delta_{1}^{2} d P<C \int \Delta d P$.

We next define holomorphic partitions of unity: Let $\Psi$ be the outer function so that $|\Psi|=\Delta_{1}$. Consider now the stopping times $\tau_{0}=0$ and

$$
\tau_{i}:=\inf \left\{t>\tau_{i-1}:\left|E\left(\Psi \mid \mathcal{F}_{t}\right)\right|>M^{i}\right\}
$$


to define $\Psi_{i}:=E\left(\Psi \mid \mathcal{F}_{\tau_{i}}\right)$ and $d_{i}:=\Psi_{i+1}-\Psi_{i}$, elements of $H^{\infty}(\Omega)$ for which obviously the identity

$$
1=\frac{E(\Psi)}{\Psi}+\sum_{i=0}^{\infty} \frac{d_{i}}{\Psi}
$$

holds. The summands of the above expression will be our choice of $\theta_{i}:$ Indeed we define $\theta_{-1}:=\frac{E(\Psi)}{\Psi}$ and $\theta_{i}:=\frac{d_{i}}{\Psi}$ for $i=0,1,2, \ldots$ We obtain from

$$
\left|d_{i}\right| \leq 2 A(\Psi) \leq 2 A(|\Psi|)=A\left(\Delta_{1}\right) \leq 3 C \Delta_{1}=3 C|\Psi|
$$

that

$$
\left\|\theta_{i}\right\|_{\infty} \leq 3 C \text {. }
$$

Havin's lemma allows us to truncate the above partition of unity: We apply it to sets $E_{i}:=\left\{\Psi^{*}>M^{i}\right\}$ and denote the resulting functions by $\alpha_{i}, \beta_{i}$. Then, following Bourgain, we define for $i=$ $-1,0,1, \ldots$

$$
w_{i}:=5 \alpha_{i} \prod_{s=8}^{\infty} \beta_{i+s} .
$$

Verification of property (5). We first eliminate the weight $\Delta$ :

$$
\begin{aligned}
\int\left|1-\sum_{i=-1}^{\infty} \theta_{i} w_{i}^{4}\right| \Delta d P & =\int\left|\sum_{i=-1}^{\infty} d_{i}\left(1-w_{i}^{4}\right)\right| \Delta^{\frac{1}{2}} d P \\
& \leq\left(\int\left|\sum_{i=-1}^{\infty} d_{i}\left(1-w_{i}^{4}\right)\right|^{2} d P\right)^{\frac{1}{2}}\left(\int \Delta\right)^{\frac{1}{2}}
\end{aligned}
$$

The martingale differences $d_{i}$ are supported on $E_{i}$ and bounded by $M^{i+1}$. Moreover, $\left|w_{i}\right|<5 \epsilon$ on $E_{i+1}$ and $E_{i+s} \subset E_{i}$ for any $s$. Therefore, we obtain a domination by:

$$
C\left(\sum_{i=-1}^{\infty} M^{(i+1) 2} \int_{E_{i}}\left|\left(1-w_{i}^{4}\right)\right|^{2} d P\right)^{\frac{1}{2}}
$$

Using the inequality

$$
\left|1-\prod z_{i}\right| \leq \sum\left|1-z_{i}\right|
$$


which holds for complex numbers in the closed unit disc, we get the following upper bound for the square of the above sum of integrals:

$$
\sum_{i} \int_{E_{i}}\left|\left(1-5 \alpha_{i}\right)\right| M^{2(i+1)}+\sum_{s>8} M^{(i+1) 2} \int_{E_{i}}\left|\left(1-\beta_{i+s}\right)\right| d P .
$$

Invoking the estimates from Havin's Lemma and applying CauchySchwarz' inequality give the following estimates:

$$
\epsilon M C+\log \left(\epsilon^{-1}\right) \sum_{s>8} \sum_{i=-1}^{\infty} M^{(i+1) 2} P\left(E_{i}\right)^{\frac{1}{2}} P\left(E_{i+s}\right)^{\frac{1}{2}} .
$$

Again by Cauchy-Schwarz we dominate the above sum by:

$$
\begin{array}{r}
\epsilon M C+\log \left(\epsilon^{-1}\right) \sum_{s>8} M^{2-s}\left(\sum_{i=-1}^{\infty} M^{2 i} P\left(E_{i}\right)\right)^{\frac{1}{2}}\left(\sum_{i=-1}^{\infty} M^{2(i+s)} P\left(E_{i+s}\right)\right)^{\frac{1}{2}} \\
\leq \epsilon M C+\log \left(\epsilon^{-1}\right) \sum_{s>8} M^{2-s} C
\end{array}
$$

This is what we want if $\epsilon$ is chosen of order $M^{-2}$ and $M:=\delta^{-1}$. Havin's lemma, repeatedly applied, gives properties (4) and (3), i.e.,

1. $\sum M^{2 i}\left\|w_{i}^{2}\right\|_{1} \leq \sum M^{2 i}\left\|\alpha_{i}\right\|_{1} \leq \sum M^{2 i} P\left(E_{i}\right)|\log \epsilon|$

2. $\left|w_{i}^{2}\right| \Delta<M^{2(i+8)}$.

Verification of property (2). As $\alpha_{i}, \beta_{i}$ satisfy $\left|\alpha_{i}\right|+\left|\beta_{i}\right| \leq 1$ we obtain $\sum\left|w_{i}\right| \leq 40$ from the following elementary considerations concerning reals.

Let $s_{i}, t_{i}$ be non negative real numbers so that

$$
s_{i}+t_{i} \leq 1 \quad i \in \mathbb{N} \text {. }
$$

Then for any $n \in \mathbb{N}$

$$
\sum_{i=1}^{n} s_{i} \prod_{i<j \leq n} t_{j} \leq 1
$$

Indeed, for $n=1$ this claim is true. (Assuming that the product over the empty index set equals 1.)

Assume the claim holds for " $n$ ", then consider

$$
s_{n+1}+t_{n+1} \leq 1
$$


Multiplying $t_{n+1}$ with the LHS of the inequality gives:

$$
s_{n+1}+\left(\sum_{i=1}^{n} s_{i} \prod_{i<j \leq n} t_{j}\right) t_{n+1} \leq 1
$$

or:

$$
\sum_{i=1}^{n+1} s_{i} \prod_{i<j \leq n+1} t_{j} \leq 1
$$

(which proves the claim).

Passing to the limit we see that

$$
\sum_{i=1}^{\infty} s_{i} \prod_{i<j} t_{j} \leq 1
$$

Hence for any $K \subseteq \mathbf{N}$

$$
\sum_{i \in K} s_{i} \prod_{\substack{j>i \\ j \in K}} t_{j} \leq 1
$$

Taking arithmetic progressions we divide $\mathbf{N}$ disjointly into $K_{0}, \ldots, K_{7}$ such that $i, j \in K_{m}$ implies $|i-j| \geq 8$. For such $K$ we find $\left(0 \leq t_{i} \leq 1\right)$

$$
\sum_{i \in K} s_{i} \prod_{j \geq i+8} t_{j} \leq 1
$$

Therefore

$$
\sum_{i \in \mathbb{N}} s_{i} \prod_{j \geq i+8} t_{j} \leq 8
$$

As $\left|w_{i}\right| \leq 5 \cdot\left|\alpha_{i}\right| \prod_{j \geq i+8}\left|\beta_{j}\right|$ we obtain

$$
\sum\left|w_{j}\right| \leq 40
$$

To finish the proof of Theorem 4 it is now enough to take $c_{i}=$ $M^{2 i}$.

3. Reduction of J. Bourgain's partition of unity. To obtain J. Bourgain's original result, we lift the density $f$ from $T$ to $\Omega$ construct a new weight together with holomorphic partitions of unity there and project the solutions back to $T$. This is done by using norm-one operators

$$
M: H^{p}(T) \rightarrow H^{p}(\Omega)
$$


and

$$
N: H^{p}(\Omega) \rightarrow H^{p}(T)
$$

so that $I d=N M$, and $N(M(f) F)=f N(F)$. (For the construction of $M$ and $N$ see [V].)

Proof of Theorem 1. Apply Theorem 4 to the density $\Delta:=M f$. Let

$$
\begin{aligned}
g_{i} & :=N\left(\theta_{i}\right), \\
\tau_{i} & :=N\left(w_{i}^{2}\right)
\end{aligned}
$$

Using $\S 2$ it is easy to verify conditions (1) ...(5) of Bourgain's theorem.

4. Truncating functions in weighted $H^{p}$. Here we combine stopping times and holomorphic partitions of unity to obtain a Marcinkiewicz decomposition in weighted Hardy spaces.

Although the next theorem looks terribly complicated, it simply states that up to a (reasonable) change of density and up to a small error, interpolation is possible in weighted Hardy spaces.

THEOREM 6. For any density $\Delta$ on $\Omega$ and $\delta>0$ there exists $\phi \in H^{\infty}(\Omega)$ and a density $\Delta_{1}$ so that the following conditions hold:

1. $\|\phi\|_{\infty}<C$

2. $\Delta_{1}>\Delta$ and $\int \Delta_{1} d P<\delta^{-C} \int \Delta d P$

3. $\int|1-\phi| \Delta d P<\delta\|\Delta\|_{1}$.

4. For $q>2, f \in H^{q}\left(\Omega, \Delta_{1}\right)$ and $\lambda>0$ there exists $g \in H^{\infty}(\Omega)$ and $h \in H^{2}(\Omega, \Delta)$ satisfying
(a) $f \phi=g+h$
(b) $\|g\|_{\infty} \leq \lambda$
(c) $\int|h|^{2} \Delta d P \leq C_{q} \lambda^{2-q} \int\left|f^{q}\right| \Delta_{1} d P$.

REMARK. Condition 4(c) involves the original density $\Delta$ in the LHS and the new weight $\Delta_{1}$ in the RHS. Although weaker than Kisliakov's result, Theorem 6 suffices to deduce Bourgain's interpolation inequality for $q$-summing operators. 
Proof. Let $w_{i} \in H^{\infty}(\Omega)$ and $\theta_{i} \in H^{\infty}(\Omega)$ be given by Theorem 4 . Then we define:

$$
\begin{aligned}
\phi & :=\sum \theta_{i} w_{i}^{4} \\
\Delta_{1} & :=\Delta+\sum c_{i}\left|w_{i}^{2}\right| \\
f_{i} & :=w_{i}^{2} f .
\end{aligned}
$$

Now we use the stopping time

$$
\tau_{j}:=\inf \left\{t:\left|E\left(f_{j} \mid \mathcal{F}_{t}\right)\right|>\lambda\right\}
$$

to define $g_{j}:=E\left(f_{j} \mid \mathcal{F}_{\tau_{j}}\right)$ and $h_{j}:=f_{j}-g_{j}$. By the stability property of holomorphic martingales these functions are certainly holomorphic and satisfy

1. $\left\|g_{j}\right\|_{\infty} \leq \lambda$

2. $\int\left|h_{j}\right|^{2} d P \leq 4 \int_{\left\{\left|f_{j}^{*}\right|>\lambda\right\}}\left|f_{j}\right|^{2} d P$.

Now using partitions of unity we glue these partial solutions together

$$
g:=\sum g_{j} w_{j}^{2} \theta_{j}
$$

and

$$
h:=\sum h_{j} w_{j}^{2} \theta_{j}
$$

Then clearly

$$
g+h=\sum\left(g_{j}+h_{j}\right) w_{j}^{2} \theta_{j}=f \phi
$$

and

$$
\|g\|_{\infty}<C \sup _{j}\left\|g_{j}\right\|_{\infty}\left\|\sum\left|w_{j}\right|\right\|_{\infty}<\lambda C^{\prime} .
$$

The estimate for $\int|h|^{2} \Delta d P$ follows a well established pattern, which has been carefully presented in the central chapter of Wojtaszczyk's book. See [W, Ch III.I].

Cauchy-Schwartz inequality (for the sequence space $l^{2}$ ) and property (2) of Theorem 4 imply that:

$$
\int|h|^{2} \Delta d P \leq C \sum \int\left|h_{j}\right|^{2}\left|w_{j}\right|^{2} \Delta d P .
$$

The last sum can be estimated, using the interplay between the partitions and the density, by

$$
\sum c_{j} \int\left|h_{j}\right|^{2} d P \leq \sum c_{j} \int_{\left\{\left|f_{j}^{*}\right|>\lambda\right\}}\left|f_{j}\right|^{2} d P .
$$


Then we finish the proof as follows:

$$
\begin{aligned}
\sum c_{j} \int_{\left\{f_{j}^{*}>\lambda\right\}}\left|f_{j}\right|^{2} d P & \leq \sum c_{j} \lambda^{2-q} \int\left|f_{j}^{*}\right|^{q} d P \\
& \leq C \lambda^{2-q} \sum c_{j} \int\left|f_{j}\right|^{q} d P \\
& \leq C \lambda^{2-q} \int\left(\left|f^{q}\right| \cdot \sum c_{j}\left|w_{j}\right|^{q}\right) d P \\
& \leq C \lambda^{2-q} \int|f|^{q} \Delta_{1} d P .
\end{aligned}
$$

5. Reduction of J. Bourgain's interpolation inequality. For a 2-summing operator $S$ there exists a positive probability measure on $\mathbf{T}$ so that

$$
\|S x\| \leq \pi_{2}(S)\left(\int|x| d \mu\right)^{\frac{1}{2}} \quad \text { for } x \in A .
$$

Without loss of generality we may assume that $\mu$ is absolutely continuous with respect to Lebesgue measure, i.e.,

$$
d \mu=f d t .
$$

Consequently for $b \in H^{\infty}(\Omega)$ the operator $U=S N$ satisfies

$$
\|U b\| \leq \pi_{2}(S)\left(\int|b| \Delta d P\right)^{\frac{1}{2}}
$$

where $\Delta=M f$.

Proof of Theorem 3. Let $0<\delta<1$ be given. Theorem 6 applied to the density $\Delta$ shows that $U$ can be split into $U=U_{1}+R_{1}$ so that

$$
\pi_{q}\left(U_{1}\right) \leq \delta^{-C} \pi_{2}(S)^{\frac{2}{q}}\|S\|^{1-\frac{2}{q}}
$$

and

$$
\pi_{2}\left(R_{1}\right) \leq \delta \pi_{2}(S)
$$

where $U_{1} b=U(b \phi)$ and $R_{1} b=U(b(1-\phi))$. Indeed fix $b \in H^{q}\left(\Omega, \Delta_{1}\right)$ of norm one in that space. Then according to Theorem 6 for $\lambda=$ $\|S\|^{\frac{q}{2}} \pi_{2}(S)^{-\frac{2}{q}}$ we find a Marcinkiewicz decomposition of $\phi b$ into

$$
\phi b=g+h .
$$


Therefore

$$
\begin{aligned}
\left\|U_{1} b\right\| & =\|U b \phi\| \leq\|U g\|+\|U h\| \\
& \leq\|S\|\|g\|_{\infty}+\pi_{2}(S)\left(\int|h|^{2} \Delta d P\right)^{\frac{1}{2}} \\
& \leq\|S\| \lambda+\pi_{2}(S) \lambda^{1-\frac{q}{2}} \leq\|S\|^{1-\frac{2}{q}} \pi_{2}(S)^{\frac{2}{q}} .
\end{aligned}
$$

As for the error term we have

$$
\|U(b(1-\phi))\| \leq \pi_{2}(S)\left(\int|b|^{2}|1-\phi|^{2} \Delta d P\right)^{\frac{1}{2}} .
$$

Using properties (3), (4) and (5) of Theorem 4 give

$$
\int|1-\phi|^{2} \Delta d P \leq C \int|1-\phi| \Delta d P \leq C \delta \int \Delta d P
$$

and

$$
\int \Delta_{1} d P \leq \delta^{-C} \int \Delta d P
$$

We therefore obtained the correct estimates for $U_{1}$ and $R_{1}$. To finish the proof of Theorem 3, we now iterate the above decomposition and observe that $S=U M$.

\section{REFERENCES}

[B1] J. Bourgain, New Banach space properties of the disc algebra and $H^{\infty}$, Acta. Math., 152 (1984), 1-48.

[B2] Bilinear forms on $H^{\infty}$ and bounded bianalytic functions, Trans. Amer. Math. Soc., 286 (1984), 313-337.

[C] K. Carne, The algebra of bounded holomorphic martingales, J. Funct. Anal., 45 (1982), 95-108.

[F] H. Föllmer, Stochastic holomorphy, Math. Ann., 207 (1974), 245-255.

[K1] S. Kislyakov, Absolutely summing operators on the disc algebra, St. Petersburg Math. J., 3 (1991), 705-774 .

[K2] _ Extensions of $(p, q)$ summing operator on the disc algebra with an appendix on Bourgain's analytic partition, preprint 1990.

[K3] - Truncating functions in weighted $H^{p}$ and two theorems of $J$. Bourgain, preprint Upsala University, 1989.

[M1] P.F.X. Müller, Holomorphic martingales and interpolation between Hardy spaces, J. d'Analyse Math. Jerusalem, 61 (1993), 327-337. 
[M2] , Holomorphic martingales and interpolation between Hardy spaces: The complex method, Trans. Amer. Math. Soc., 347 (1995), 1787-1792.

[P] G. Pisier, A simple proof of a theorem of J. Bourgain, Michigan Math. J., 39 (1992), 475-484.

[V] N. Varopoulos, Helson Szego Theorem $A_{p}$ functions for Brownian motion and several variables, J. Funct. Anal., 39 (1980), 85-121.

[W] P. Wojtaszczyk, Banach spaces for analysts, Cambridge Univ. Press 1991.

Received November 12, 1992. The author was supported by FFWF Pr.Nr. JP 90061.

\section{J. KEPLER UNIVERSITÄT}

LINZ

Austria 



\title{
PACIFIC JOURNAL OF MATHEMATICS
}

Founded by E. F. Beckenbach (1906-1982) and F. Wolf (1904-1989)

\section{EDITORS}

Sun-Yung Alice Chang (Managing Editor)

University of California

Los Angeles, CA 90095-1555

pacific@math.ucla.edu

F. Michael Christ

University of California

Los Angeles, CA 90095-1555

christ@math.ucla.edu

Thomas Enright

University of California

San Diego, La Jolla, CA 92093

tenright@ucsd.edu

Nicholas Ercolani

University of Arizona

Tucson, AZ 85721

ercolani@math.arizona.edu
Robert Finn

Stanford University

Stanford, CA 94305

finn@gauss.stanford.edu

Vaughan F. R. Jones

University of California

Berkeley, CA 94720

vfr@math.berkeley.edu

Steven Kerckhoff

Stanford University

Stanford, CA 94305

spk@gauss.stanford.edu
Martin Scharlemann

University of California

Santa Barbara, CA 93106

mgscharl@math.ucsb.edu

\section{Gang Tian}

Courant Institute

New York University

New York, NY 10012-1100

tiang@taotao.cims.nyu.edu

V. S. Varadarajan

University of California

Los Angeles, CA 90095-1555

vsv@math.ucla.edu

\section{SUPPORTING INSTITUTIONS}

\section{CALIFORNIA INSTITUTE OF TECHNOLOGY \\ NEW MEXICO STATE UNIVERSITY \\ OREGON STATE UNIVERSITY \\ STANFORD UNIVERSITY \\ UNIVERSITY OF ARIZONA \\ UNIVERSITY OF BRITISH COLUMBIA \\ UNIVERSITY OF CALIFORNIA UNIVERSITY OF HAWAII}

\author{
UNIVERSITY OF MONTANA \\ UNIVERSITY OF NEVADA, RENO \\ UNIVERSITY OF OREGON \\ UNIVERSITY OF SOUTHERN CALIFORNIA \\ UNIVERSITY OF UTAH \\ UNIVERSITY OF WASHINGTON \\ WASHINGTON STATE UNIVERSITY
}

The supporting Institutions listed above contribute to the cost of publication of this Journal, but they are not owners or publishers and have no responsibility for its contents or policies.

Manuscripts must be prepared in accordance with the instructions provided on the inside back cover.

The Pacific Journal of Mathematics (ISSN 0030-8730) is published monthly except for July and August. Regular subscription rate: $\$ 215.00$ a year (10 issues). Special rate: $\$ 108.00$ a year to individual members of supporting institutions.

Subscriptions, orders for back issues published within the last three years, and changes of subscribers address should be sent to Pacific Journal of Mathematics, P.O. Box 4163, Berkeley, CA 94704-0163, U.S.A. Prior back issues are obtainable from Kraus Periodicals Co., Route 100, Millwood, NY 10546.

The Pacific Journal of Mathematics at the University of California, c/o Department of Mathematics, 981 Evans Hall, Berkeley, CA 94720 (ISSN 0030-8730) is published monthly except for July and August. Second-class postage paid at Berkeley, CA 94704, and additional mailing offices. POSTMASTER: send address changes to Pacific Journal of Mathematics, P.O. Box 6143, Berkeley, CA 94704-0163.

\author{
PUBLISHED BY PACIFIC JOURNAL OF MATHEMATICS at University of California, \\ Berkeley, CA 94720, A NON-PROFIT CORPORATION \\ This publication was typeset using AMS-LATEX, \\ the American Mathematical Society's TEX macro system. \\ Copyright (C) 1995 by Pacific Journal of Mathematics
}




\section{PACIFIC JOURNAL OF MATHEMATICS}

Volume 169 No. $1 \quad$ May 1995

Minimal sets of periods for torus maps via Nielsen numbers

Lluís AlsedÀ, StewART BALdWin, JAUME Llibre,

RICHARD SWANSON and WIESLAW SZLENK

Diagonalizing Hilbert cusp forms

Timothy ATWILL

A splitting criterion for rank 2 vector bundles on $\mathbf{P}^{n}$

EDOARDO BALLICO

Controlling Tietze-Urysohn extensions

MARC FRANTZ

Length of Julia curves

DAVID H. HAMILTON

On the uniqueness of capillary surfaces over an infinite strip

JENN-FANG HWANG

Volume estimates for log-concave densities with application to iterated 107 convolutions

MARIUS JUNGE

A reflection principle in complex space for a class of hypersurfaces and mappings

FRANCINE ANTOINETTE MEYLAN

Jean Bourgain's analytic partition of unity via holomorphic martingales 161

PAUL F.X. MÜLLER

Characters of Brauer's centralizer algebras 